\title{
PERFIL SOCIODEMOGRÁFICO E CLÍNICO DE PACIENTES EM PÓS-OPERATÓRIO DE CONFECÇÃO DE ESTOMAS INTESTINAIS DE ELIMINAÇÃO
}

\author{
SOCIODEMOGRAPHIC AND CLINICAL PROFILE OF \\ PATIENTS IN POSTOPERATIVE RECOVERY FROM INTESTINAL \\ STOMA CREATION
}

\author{
PERFIL SOCIODEMOGRÁFICO Y CLÍNICO DE PACIENTES EN \\ POSOPERATORIO DE PRODUCCIÓN DE ESTOMAS INTESTINALES
}

\author{
Marcelo Victor Freitas Nascimento* \\ Samuel Oliveira da Vera ** \\ Maria Caroline Rodrigues Silva ${ }^{* *}$ \\ Fernanda Ferreira de Morais ${ }^{* * * *}$ \\ Elaine Maria Leite Rangel Andrade ${ }^{* * * *}$ \\ Sarah Nilkece Mesquita Araújo Nogueira Bastos ${ }^{* * * * *}$
}

\section{RESUMO}

\begin{abstract}
Objetivo: Analisar o perfil sociodemográfico e clínico dos pacientes no pós-operatório de confecção de estoma intestinal. Material e método: Estudo de abordagem quantitativa, descritiva, realizado no período de agosto a outubro de 2015, em um serviço filantrópico de referência em oncologia, localizado em Teresina, capital da província do Piauí, Brasil. Um questionário sociodemográfico, clínico e um exame físico com ênfase na avaliação do abdome e do estoma foram aplicados em 56 pacientes. Resultados: Perfil sociodemográfico: idade média de 57,75 anos, predominantemente mulheres $(67,9 \%)$, do interior do Piauí $(62,5 \%)$, residentes em áreas urbanas $(69,6 \%)$, raça mulata $(53,6 \%)$, religião católica $(78,6 \%)$, com ensino fundamental incompleto $(37,5 \%)$ e renda até um salário mínimo (67,9\%). Perfil clínico: 42,9\% tinham câncer de reto como diagnóstico médico, 85,7\% tinham colostomia, em $76,8 \%$ a cirurgia era eletiva e não demarcada $(57,1 \%)$, com equipamento coletor de 1 peça $(51,8 \%)$, boa fixação da equipe de coleta $(87,5 \%)$ e estoma com boa função $(89,3 \%)$. Conclusão: A assistência ao estomizado deve estar embasada no conhecimento científico do processo de enfermagem e identificar as principais necessidades de cada paciente, de acordo com seus perfis sociodemográficos e clínicos.
\end{abstract}

Palavras chave: Perfil sociodemográfico; Perfil clínico; Estomas cirúrgicos; Período pós-operatório; Enfermagem Perioperatória.

\footnotetext{
ABSTRACT

Objective: To evaluate the sociodemographic and clinical profile of patients in the postoperative period following intestinal stoma surgery. Material and method: Quantitative study, with a descriptive approach carried

*Enfermeiro, Especialista, Universidade Federal do Piauí. Email: marcelovyctor16@hotmail.com

** Enfermeiro, Especialista, Universidade Federal do Piauí. Email: Oliveira-samuel@outlook.com

*** Enfermeira, Especialista, Associação de Ensino Superior do Piauí. Email: karol_rodrigues16@hotmail.com

${ }^{* * * *}$ Enfermeira, Associação de Ensino Superior do Piauí. Email: fern.ada.moraes@hotmail.com

${ }^{* * * * *}$ Enfermeira, Doutora, Universidade Federal do Piauí. Email: elairgel@gmail.com

${ }^{* * * * * *}$ Enfermeira, Doutora, Universidade Federal do Piauí. Email: sarahnilkece@hotmail.com
} 
out between August and October 2015, in a philanthropic service of reference in an oncology unit, located in Teresina, capital of the province of Piauí, Brazil. A sociodemographic and clinical questionnaire plus a physical examination with emphasis on the evaluation of the abdomen and stoma were applied to 56 patients. Results: Sociodemographic profile: average age of 57.75 years, mostly women (67.9\%), from the interior of Piauí (62.5\%), who live in urban areas (69.6\%), of mulatto ethnicity (53.6\%), Catholic religion (78.6\%), with incomplete primary education (37.5\%) and an income up to minimum wage (67.9\%). Clinical profile: $42.9 \%$ had rectal cancer as a medical diagnosis, $85.7 \%$ had colostomy; the surgery was elective in $76.8 \%$ and not demarcated $(57.1 \%)$, with a one-piece collector bag system (51.8\%), good fixation of the collector bag ( $87.5 \%)$ and stoma with good function $(89.3 \%)$. Conclusion: The care of patients with stomas should be based on scientific knowledge of the nursing process, and the main needs of each patient should be identified based on their sociodemographic and clinical profiles.

Key words: Sociodemographic profile; Clinical profile; Surgical stomas; Postoperative period; Perioperative Nursing.

\section{RESUMEN}

Objetivo: Evaluar el perfil sociodemográfico y clínico de los pacientes en posoperatorio mediato de estoma intestinal. Material y método: Estudio de abordaje cuantitativo, descriptivo realizado entre agosto a octubre de 2015, en un servicio filantrópico de referencia en oncología, ubicado en Teresina, capital de la provincia de Piauí, Brasil. A 56 pacientes se les aplicó un cuestionario sociodemográfico y clínico más un examen físico con énfasis en la evaluación del abdomen y el estoma. Resultados: Perfíl sociodemográfico: edad media de 57,75 años, en su mayoría mujeres (67,9\%), del interior del Piauí (62,5\%), que viven en zonas urbanas (69,6\%), de raza mulata $(53,6 \%)$, religión católica $(78,6 \%)$, con escolaridad primaria incompleta $(37,5 \%)$ y un ingreso de hasta un salario mínimo (67,9\%). Perfil clínico: el 42,9\% tenía cáncer del recto como un diagnóstico médico, el $85,7 \%$ tenía colostomía, en el $76,8 \%$ la cirugía fue electiva y no demarcada $(57,1 \%)$, con equipos colectores de 1 pieza (51,8\%), buena fijación del equipo recolector $(87,5 \%)$ y estoma con buena función $(89,3 \%)$. Conclusión: La atención al paciente estomizado debe basarse en el conocimiento científico del proceso de enfermería e identificar las principales necesidades de cada paciente en función de sus perfiles sociodemográficos y clínicos.

Palabras clave: Perfil sociodemográfico; Perfil clínico; Estomas quirúrgicos; Período posoperatorio; Enfermería Perioperatoria.

Fecha recepción: 16/12/2017 Fecha aceptación: 21/06/2018

\section{INTRODUÇÃO}

Denomina-se estomia a exteriorização de víscera oca do organismo, mediante um procedimento cirúrgico, a fim de desviar as eliminações, para tratar patologias do aparelho gastrointestinal, respiratório ou urinário, em decorrência de doenças inflamatórias, anomalias congênitas, aos traumatismos e, principalmente, às neoplasias ${ }^{(1)}$.

A nomeação da estomia varia de acordo com o segmento corporal afetado. As estomias intes- tinais são as jejunostomias, ileostomias e colostomias, se forem confeccionadas no jejuno, íleo e cólon, respectivamente. As ileostomias e colostomias são consideradas estomias intestinais de eliminação (EIE), por desviarem o trânsito fecal para o meio externo. Estas por sua vez, são classificadas quanto ao tempo de permanência, como definitivas ou temporárias ${ }^{(2)}$. Suas características físicas variam quanto ao tipo, localização, tamanho, forma, superfície, contorno e protusão, que podem variar de acordo com a técnica cirúrgica utilizada, o segmento exteriorizado, a causa bási- 
ca e o tempo de permanência ${ }^{(3)}$.

Com base nessa vertente, estudos abrangendo pacientes com EIE são extremamente importantes, pois o número de estomizados aumenta continuamente. Segundo o Instituto Nacional do Câncer, estima-se 15.070 casos novos de câncer de cólon e reto em homens e 17.530 mulheres. Mundialmente, uma em cada 10 mil pessoas é estomizada. Evidencia-se que o quantitativo de pessoas estomizadas no Brasil corresponde a 33.864, no entanto, faz-se necessário ressaltar que este número é ainda maior, devido a existência de subnotificações ${ }^{(4)}$. No Brasil, 1,4 milhões de pessoas fazem uso de equipamento coletor em decorrência de EIE e urinárias ${ }^{(5)}$.

Os dados aumentam, em decorrência, principalmente, do incremento do número de casos de câncer colorretal. Estima-se, para o Brasil, biênio 2018-2019, a ocorrência de 600 mil casos novos de câncer, para cada ano. Excetuando-se o câncer de pele não melanoma (cerca de 170 mil casos novos), ocorrerão 420 mil casos novos de câncer, aponta-se a ocorrência de 640 mil casos novos. Essas estimativas refletem o perfil de um país que possui os cânceres de próstata, pulmão, mama feminina e cólon e reto entre os mais incidentes, entretanto ainda apresenta altas taxas para os cânceres do colo do útero, estômago e esôfago ${ }^{(6)}$. Nestes casos dos canceres do cólon e reto, as EIE são confeccionados ainda como medida paliativa para prorrogação da vida dos indivíduos e das causas externas, como traumas e violências, estão presentes, principalmente na população jo$\operatorname{vem}^{(2-5)}$.

A confecção de uma EIE pode acarretar diversas complicações físicas e emocionais na vida do indivíduo estomizado. Uma estomia, como parte de cura para um câncer, traz mudanças nos hábitos de vida, alterações fisiológicas e psicológicas para o paciente e família e pode significar muito na vida do paciente e da família ${ }^{(7)}$. Ao se deparar com o estoma, o paciente passa a lidar com esta nova realidade quando são suscitados vários sentimentos, reações e comportamentos, diferentes e individuais. Assim, a presença de um estoma pode resultar em uma depressão psico- lógica, contribuindo para uma diminuição ou deteriorização da sua qualidade de vida, pois o estomizado tende a se sentir estigmatizado, por julgar-se diferente, ou seja, por não apresentar as características e os atributos considerados normais pela sociedade ${ }^{(8)}$.

Nesse sentindo, é importante o papel do enfermeiro no pós-operatório (PO) mediato para fornecer apoio emocional ao paciente, estar atento à integridade cutânea, ensinar o paciente a trocar corretamente os dispositivos, identificar se as necessidades hídricas e nutricionais estão sendo sanadas e ficar atento a sinais de complicações.

Sendo assim, sentiu-se a necessidade de aprofundar mais nesta área, com o foco voltado para o perfil sociodemográfico e clinico de pacientes em PO mediato de confecção de EIE. A caracterização desses pacientes, visam um melhor atendimento, em todos os níveis de complexidade, promovendo principalmente o papel e o compromisso do enfermeiro na busca de um melhor atendimento e a possibilidade da realização de intervenções mais eficazes.

Assim, este estudo tem como objetivo analisar o perfil sociodemográfico e clínico de pacientes em PO mediato de confecção de EIE.

\section{MATERIAL E METODO}

Tipo de estudo: Trata-se de um estudo com abordagem quantitativa, descritivo e transversal.

Local de estudo e unidade de análise: O estudo desenvolveu-se em um Hospital filantrópico situado em Teresina, capital do estado do Piauí, Brasil, que é um serviço de referência em alta complexidade na saúde, com ênfase em oncologia, ensino e pesquisa. Dispõe de cerca de 380 leitos, sendo a clínica cirúrgica do referido hospital composta de 130 leitos, e com 142 profissionais disponíveis para a mesma, sendo 14 enfermeiros e 128 técnicos de enfermagem. A população do estudo compôs-se de todos os pacientes admitidos nas clínicas cirúrgicas do hospital e que 
se encontravam em PO mediato de confecção de EIE, no período da coleta de dados que ocorreu nos meses de agosto a outubro de 2015.

Muestra: A amostragem do tipo não-probabilística por conveniência, foi formada por 56 pacientes, que atendiam aos critérios de inclusão pré-estabelecidos: idade superior a 18 anos, estar em PO mediato de confecção de EIE ou que estivesse internado para tratamento de complicações relacionadas à EIE. Foram excluídos da amostra crianças e pacientes com outras estomias, que não fossem intestinais de eliminação.

Coleta de dados: Inicialmente foi aplicado um questionário contendo variáveis sociodemográficas e clínicas, bem como antecedentes pessoais e familiares, hábitos de vida, caracterizando a fase da anamnese do histórico de enfermagem. Em seguida, realizou-se o exame físico crânio-caudal, seguindo as técnicas semiológicas de inspeção, palpação, percussão e ausculta ${ }^{(9)}$, com ênfase na avaliação do abdome e da EIE.

Processamento e análise. Os dados obtidos foram armazenados em um banco eletrônico criado no programa Excel 2010 (Windows 7) e posteriormente por meio de estatística descritiva (frequência absoluta, frequência percentual e média) foram analisados com meio do Statistical Package for the Social Sciences- SPSS (versão 17.0). Para verificar normalidade das variáveis, utilizou-se o teste de Kolmogorov-Smirnov.

Salvaguardas éticas: Atendendo aos aspectos éticos que regem as pesquisas que envolvem seres humanos, preconizadas pela Resolução no 466/12 do Conselho Nacional de Saúde ${ }^{(10)}$, foi elaborado um termo de consentimento livre e esclarecido (TCLE), com o intuito de preservar a privacidade e confidencialidade dos participantes. O projeto foi submetido ao Comitê de Ética em Pesquisa da Universidade Paulista-UNIP e aprovado com o número do Protocolo: 45430515.0.0000.5512.

\section{RESULTADOS}

Perfil Sociodemográfico dos pacientes em pósoperatório de confecção de EIE (Tabela 1). A amostra deste estudo foi constituída por $67,9 \%$ pacientes do sexo feminino. A faixa etária predominante foi a de 50 a 69 anos $(41,1 \%)$. Em relação à procedência, 62,5\% eram do interior do Piauí e 69,6\% residiam na capital de Teresina-PI, zona urbana. A maioria era de cor parda $(53,6 \%)$ e da religião católica $(78,6 \%)$. Observou-se que $33,9 \%$ possuíam outros tipos de profissões (professores, comerciantes, enfermeiros, nutricionista) e $67,9 \%$ afirmou ter renda mensal de até um salário mínimo e que a maioria $(73,3 \%)$ estudou até o ensino fundamental incompleto $(37,5 \%)$.

Perfil Clínico dos pacientes com estomas intestinais (Gráfico 1). No grupo investigado observou-se que do total pacientes, $42,9 \%$ tinham como diagnóstico médico a Neoplasia do reto. No tocante ao tipo de EIE, percebeu-se o predomínio de $85,7 \%$ de colostomias e no que diz respeito ao tipo de cirurgia realizada, $76,8 \%$ foram eletivas, sendo importante ressaltar que do total da amostra, 57,1\% não foram demarcadas previamente (Tabela 2). Identificou-se, ainda, a presença de comorbidades em 67,8\% dos estomizados, entre as quais se destacou vários outros tipos citados (33,9\%). Quanto aos antecedentes familiares, 41,1\% relataram algum caso de câncer na família (Tabela 3). Outra variável avaliada foi equipamento coletor utilizado. Para tal, consideraram-se o tipo de dispositivo em uso e a área para fixação do dispositivo. A maioria dos pacientes fazia uso do equipamento de uma peça $(51,8 \%)$. Em relação à fixação do equipamento, $87,5 \%$ relataram ter uma boa adesão do dispositivo à pele e que também ainda $89,3 \%$ possuiam uma EIE funcionante (Tabela 4). No que se refere às queixas relativas ao procedimento de confecção de EIE mais comuns, a cólica intestinal $(66,1 \%)$ predomina (Tabela 5). 
Tabela 1. Perfil sociodemográfico dos pacientes com EIE (n=56). Teresina-PI, 2015.

\begin{tabular}{|c|c|c|c|}
\hline Variáveis & & $\mathbf{N}$ & $\%$ \\
\hline \multirow[t]{2}{*}{ Sexo } & Feminino & 38 & 67,9 \\
\hline & Masculino & 18 & 32,1 \\
\hline \multirow[t]{3}{*}{ Faixa etária* } & De 29 a 49 anos & 19 & 33,9 \\
\hline & De 50 a 69 anos & 23 & 41,1 \\
\hline & Igual ou maior que 70 anos & 14 & 25 \\
\hline \multirow[t]{4}{*}{ Procedência } & Teresina & 17 & 30,4 \\
\hline & Interior do Piauí & 35 & 62,5 \\
\hline & Estado do Maranhão & 3 & 5,4 \\
\hline & Outros & 1 & 1,8 \\
\hline \multirow[t]{3}{*}{ Residência } & Zona Rural & 16 & 28,6 \\
\hline & Zona Urbana & 39 & 69,6 \\
\hline & Branca & 14 & 25 \\
\hline \multirow[t]{4}{*}{ Cor } & Negro & 7 & 12,5 \\
\hline & Amarelo & 5 & 8,9 \\
\hline & Parda & 30 & 53,6 \\
\hline & Católica & 44 & 78,6 \\
\hline \multirow[t]{3}{*}{ Religião } & Evangélica & 12 & 21,4 \\
\hline & Desempregado & 5 & 8,9 \\
\hline & Aposentado & 12 & 21,4 \\
\hline \multirow[t]{3}{*}{ Profissão } & Lavrador & 14 & 25 \\
\hline & Doméstica & 6 & 10,7 \\
\hline & Outros & 19 & 33,9 \\
\hline \multirow[t]{3}{*}{ Renda } & Não assalariado & 6 & 10,7 \\
\hline & Até 1 salário mínimo & 38 & 67,9 \\
\hline & De 2 a 4 salários mínimos & 11 & 19,6 \\
\hline \multirow[t]{7}{*}{ Escolaridade } & Não alfabetizado & 15 & 26,8 \\
\hline & Ens. Fundamental Incompleto & 21 & 37,5 \\
\hline & Ens. Fundamental completo & 10 & 17,9 \\
\hline & Ens. Médio completo & 7 & 12,5 \\
\hline & Ens. Superior incompleto & 2 & 3,6 \\
\hline & Ens. Superior completo & 1 & 1,8 \\
\hline & TOTAL & 56 & 100 \\
\hline
\end{tabular}

*Faixa etária: Média: 57,75; Desvio padrão: 14,29; Mín-Max: 20-83. Intervalo de confiança (IC): 95\%.

1 salario mínimo $=257,72$ USD. 
Gráfico 1. Perfil clínico dos pacientes com estomas intestinais (n=56). Teresina-PI, 2015.

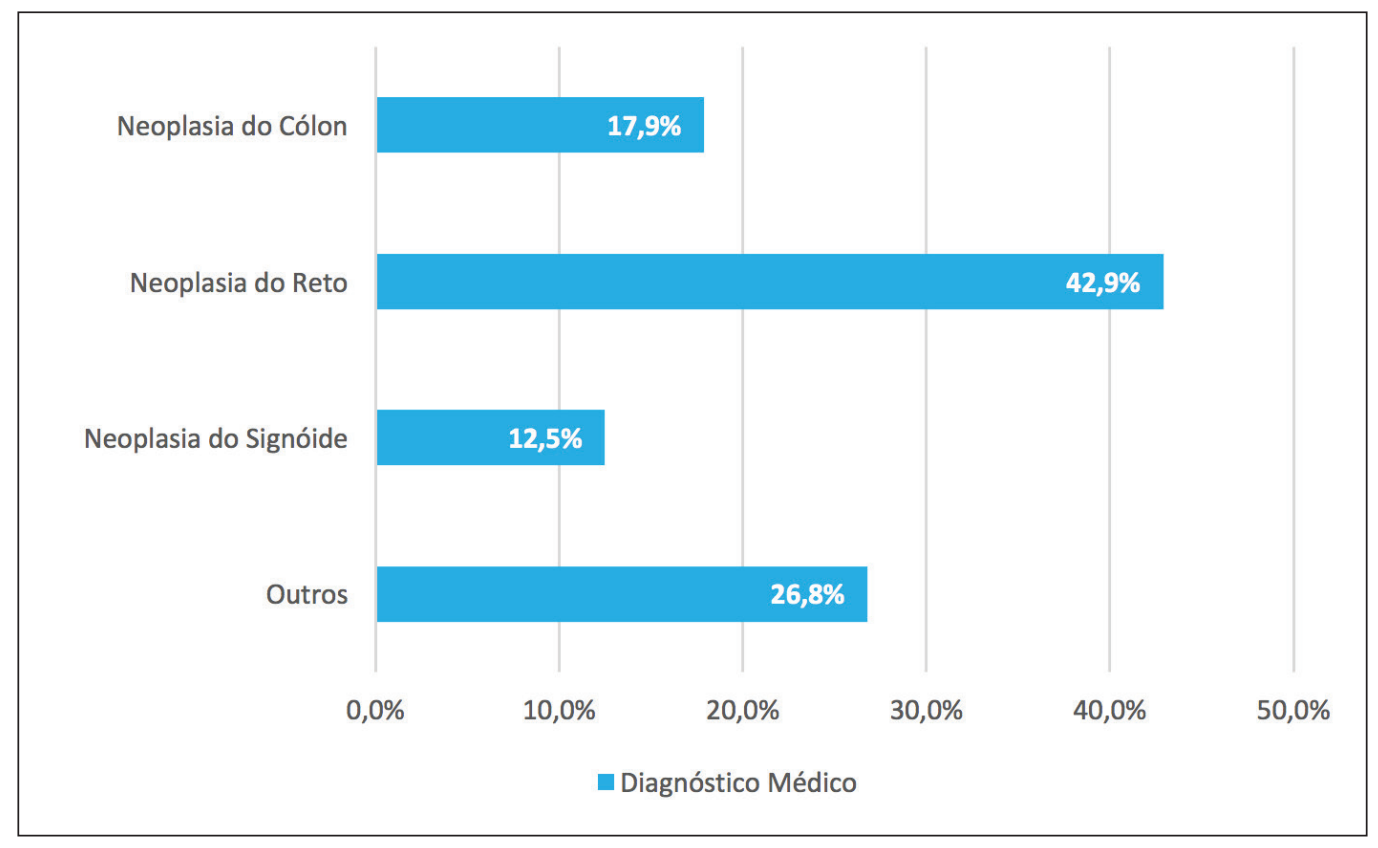

Tabela 2. Distribuição dos estomizados segundo o tipo de ostomia tipo de cirurgia e demarcação do estoma ( $\mathrm{n}=56)$. Teresina-PI, 2015.

\begin{tabular}{llcc}
\hline Variáveis & & N & \% \\
\hline Tipo de ostomia & Colostomia & 48 & 85,7 \\
& Ileostomia & 8 & 14,3 \\
\multirow{2}{*}{ Tipo de cirurgia } & Eletiva & 43 & 76,8 \\
& Urgência & 13 & 23,2 \\
\multirow{2}{*}{ Demarcada } & Não & & \\
& Sim & 32 & 57,1 \\
& Total & 24 & 42,9 \\
\hline
\end{tabular}


Tabela 3. Distribuição dos estomizados segundo a presença de comorbidades e antecedentes familiares $(\mathrm{n}=56)$. Teresina-PI, 2015.

\begin{tabular}{llcc}
\hline Variáveis & & N & $\%$ \\
\hline Comorbidades & Outras & 19 & 33,9 \\
& Nenhuma & 18 & 32,1 \\
& Hipertensão & 14 & 25 \\
& Diabetes & 5 & 8,9 \\
\multirow{3}{*}{ Antecedentes familiares } & & & \\
& Doenças cancerígenas & 23 & 41,1 \\
& Nenhuma & 14 & 22 \\
& Doenças cardiovasculares & 10 & 17,9 \\
& Outros & 9 & 16,1 \\
\hline & Total & 56 & 100 \\
\hline
\end{tabular}

Tabela 4. Distribuição dos estomizados segundo o tipo de dispositivo em uso, fixação do equipamento coletor e funcionalidade do EIE ( $\mathrm{n}=56)$. Teresina-PI, 2015.

\begin{tabular}{|c|c|c|c|}
\hline Variáveis & & $\mathbf{N}$ & $\%$ \\
\hline \multirow[t]{4}{*}{ Tipo de equipamento coletor } & 1 peça & 29 & 51,8 \\
\hline & Descartáveis & 18 & 32,1 \\
\hline & 2 peças & 8 & 14,3 \\
\hline & Protetoras & 1 & 1,8 \\
\hline \multirow[t]{2}{*}{ Fixação do equipamento coletor } & Sim & 49 & 87,5 \\
\hline & Não & 7 & 12,5 \\
\hline \multirow[t]{3}{*}{ Funcionamento da $\mathrm{EIE}^{\star}$} & Sim & 50 & 89,3 \\
\hline & Não & 6 & 10,7 \\
\hline & Total & 56 & 100 \\
\hline
\end{tabular}

${ }^{\star}$ Estomia intestinal de eliminação. 
Tabela 5. Distribuição percentual dos participantes segundo manifestações clínicas ( $\mathrm{n}=56)$. TeresinaPI, 2015.

\begin{tabular}{lcc}
\hline Tipo de manifestação & \multicolumn{2}{c}{ Presença da manifestação clínica } \\
\hline & Sim & Não \\
\cline { 2 - 3 } & $\mathbf{n}(\%)$ & $\mathbf{n}(\%)$ \\
\hline Cólica intestinal & $37(66,1)$ & $19(33,9)$ \\
Constipação & $32(57,1)$ & $24(42,9)$ \\
Dor & $22(39,3)$ & $34(60,7)$ \\
Outros & $17(30,4)$ & $39(69,6)$ \\
Melena & $14(25,0)$ & $42(75,0)$ \\
Diarreia & $5(8,9)$ & $51(91,1)$ \\
\hline
\end{tabular}

*Valores superiores ao número de pacientes que estavam em pós-operatório de EIE ( $\mathrm{n}=56)$, pois alguns pacientes apresentaram mais de uma manifestação clínica.

\section{DISCUSSÃO}

Com respeito ao perfil sociodemográfico dos pacientes em pós-operatório de confecção de EIE, houve predominância dos entrevistados do sexo feminino. Corroborando com este achado, as Estatísticas do INCA revelam uma incidência de 3.330 casos novos de câncer em mulheres, contra 2.830 casos em homens em 2012 no Piauí. Este padrão de incidência superior feminina se repete na região Nordeste e no Brasil consolidado(11, 12). O predomínio de mulheres pode estar relacionado às características da doença de base, uma vez que o Instituto Nacional do Câncer (INCA) informou que no período de 1995 a 1999(12) o câncer de colón e reto se constituiu como a quarta causa de morte entre mulheres por câncer no país, sendo superposto apenas para os cânceres de mama, pulmões e estomago ${ }^{(3)}$.

Contrariando os dados encontrados neste presente estudo, observou-se em estimativas mundial no ano de 2012, que o câncer de colo e reto são o terceiro tipo de câncer que mais acometeu homens, com 746 mil novos casos (10\% do total dos cânceres), e nas mulheres como o segundo, com 614 mil novos casos (9,2\% do total dos cânceres), de acordo com o Instituto nacio- nal do câncer (INCA), e 55\% dos casos são procedentes de países com alto índice de desenvolvimento socioeconômico ${ }^{(6,13)}$. Dados obtidos da Organização Mundial de Saúde (OMS), trazem estimativas mundiais para 2030, cerca de 21,4 milhões de casos novos de câncer e 13,2 milhões de mortes por câncer, em consequência do crescimento e do envelhecimento da população ${ }^{(14)}$.

Estudos apontam que a faixa etária mais acometida para a realização de procedimentos cirúrgicos que geram EIE é a de 58 a 78 anos, pois constitui fator de risco para o aparecimento de neoplasias e outras doenças crônicas que resultam em confecção de estomias ${ }^{(15)}$. Avaliando as faixas etárias mais atingidas observou-se o predomínio no intervalo de 50 a 69 anos, correspondendo a quase metade dos entrevistados. Contudo é visível que o aumento da incidência de estomias a partir dos 50 anos, mais com seu predomínio após os 70 anos. Dados de um outro estudo mostram estimativas para os próximos quatorze anos, que as pessoas com 65 anos ou mais representarão $70 \%$ do total de indivíduos diagnosticados com câncer ${ }^{(4,14)}$.

Vale ressaltar o precário nível socioeconômico dos participantes do estudo verificado pela baixa renda, o que pode acarretar dificuldades em adquirir os dispositivos necessários ao autocui- 
dado $^{(16)}$. No que se refere às profissões dos entrevistados, a maioria dos pacientes pertence à classe popular, caracterizada por trabalhadores que vivem em condições financeiras precárias, decorrentes da baixa escolaridade e por consequência, com acesso limitado aos serviços públicos, como educação e saúde ${ }^{(3)}$.

O baixo nível de escolaridade apresentado pelos participantes pode refletir no déficit de conhecimento sobre as medidas preventivas do câncer e as complicações das EIE, o que se torna importante às ações de educação em saúde implementadas pela equipe multiprofissional, discutindo sobre a necessidade de hábitos saudáveis de vida ${ }^{(15)}$. O uso de técnicas de educação em saúde que adotem linguagens alternativas, para o público carente, é fundamental para transformar ao paciente em um sujeito ativo do seu processo de cura ${ }^{(17)}$.

A causa desses índices baixos de nível educacional entre os estomizados relaciona-se à ainda à deficiência de conhecimentos sobre a necessidade de realizar exames de rotina para detecção precoce e prevenção do câncer, denotando a importância da atuação da equipe de saúde na educação da população ${ }^{(18)}$.

Com respeito ao perfil clínico dos pacientes com EIE, conhecer as causas básicas que motivaram as cirurgias e especificidades relacionadas às EIE são aspectos importantes a considerar no planejamento da assistência de enfermagem, principalmente no que concerne ao autocuidado. Assim, entre as causas para confecção das EIE predominaram as neoplasias do reto e sigmóide.

No Brasil ainda são poucos os estudos quantitativos sobre o perfil clínico de pessoas com EIE. No Piauí, a realidade não é diferente, pois foram localizadas no estado apenas duas publicações relacionadas ao perfil de pessoas com estomas de eliminação intestinal, todos se restringem àqueles submetidos a internação hospitalar ${ }^{(19,20)}$.

Os resultados da pesquisa revelam que o perfil clínico dos estomizados corrobora com os achados obtidos em outros estudos relacionados ao tema, em que tem demonstrado predominância das neoplasias malignas, principalmente o câncer colorretal, como principal diagnóstico médico com necessidade de intervenção cirúrgica com confecção de estoma intestinal de eliminação ${ }^{(21)}$. O câncer do cólon e reto é o segundo tipo de câncer mais prevalente no mundo, ocupando o terceiro lugar em termos de incidência, sendo mais elevada nos países em desenvolvimento ${ }^{(11)}$.

No que tange aos dados sobre o perfil demográfico e clínico ou de informações de associações tais como a Associação Brasileira de Ostomizados (ABRASO), International Ostomy Association (IOA) e United Ostomy Association (UOA), ao nível mundial e norte-americano (EUA e Canadá), apontam que as causas oncológicas ainda predominam na determinação de EIE, entretanto, ainda consideram-se importantes as causas inflamatórias, traumáticas e congênitas ${ }^{(22)}$.

Estudos apontam que entres as principais causas de confecção de EIE, o câncer colorretal e os traumas, abrange parcelas sociais cada vez maiores e mais jovens, sem contar que os estomas são confeccionados como medida paliativa para prorrogação da vida dos indivíduos. Acrescentando ainda a esses dados, uma outra pesquisa evidencia que a esse procedimento se atribui diversas causas, e entre as mais frequentes estão os traumas, doenças congênitas, doenças inflamatórias, tumores e câncer de intestino e bexiga ${ }^{(23,24)}$.

Além do câncer, entre as outras causas estão as inflamatórias, congênitas, traumáticas e iatrogenias. De acordo com a diversidade de idades dos estomizados participantes deste estudo, que inclui indivíduos adultos e idosos, podemos correlacionar tal fato com causas apontadas para confecção da EIE. Entre os adultos evidencia-se maior frequência de doenças inflamatórias intestinais, tais como a doença de Crohn e retocolite ulcerativa. Quanto aos idosos, verifica-se a predominância de quadros clínicos obstrutivos relacionados ao câncer colorretal e doença inflamatória intestinal como diverticulite ${ }^{(25)}$.

Neste estudo observou-se que a maioria dos pacientes tinham colostomia em relação à ileostomia, corroborando com a maioria dos estudos de caracterização clínica de pacientes com $\operatorname{EIE}^{(26,}$ 27). No decorrer dos meses de janeiro a março de 2012, várias instituições de saúde brasileiras realizaram cerca de nove mil cirurgias reparadoras 
do aparelho digestivo, destas, um terço resultou na realização de uma EIE (colostomia ou ileostomia), e parte dos pacientes foi submetido ao procedimento cirúrgico que se fez necessário o uso de equipamento coletor de efluentes ${ }^{(28)}$.

É relevante afirmar que a amostra deste trabalho foi composta basicamente por cirurgias eletivas. As cirurgias eletivas possuem um grande diferencial em relação às emergenciais, pelo fato de se ter um tempo para planejar a incisão cirúrgica, seguindo passos que são de grande importância para paciente no que diz respeito ao processo saúde-doença. Dentro desse processo, pode-se citar a importância do empoderamento do paciente em relação à cirurgia em si e também a técnica de demarcação da EIE no período pré-operatório, do qual se evita muitas complicações em $\mathrm{PO}^{(29)}$.

O conhecimento e a preparação sobre o estoma, previo do procedimento, dos pacientes com cirurgias eletivas, favorecem uma melhor adaptação ao estoma, em comparação àqueles cujo estoma é resultado de um trauma abdominal, já que neste último o caráter emergencial da cirurgia, apenas permite conhecer o que é o estoma e seu procedimento, após a cirurgia realizada ${ }^{(30)}$.

A demarcação da EIE no pré-operatório foi insuficiente neste estudo. Esses achados corroboram com estudo que observou que $60 \%$ dos estomizados referiram não terem sido demarcados anteriormente à cirurgia ${ }^{(31)}$. A demarcação do estoma constitui um procedimento fundamental a ser realizado no pré-operatório pelo enfermeiro estomaterapeuta, engajado no cuidado ao estomizado intestinal. É uma prática importante no processo de reabilitação e reflete diretamente na qualidade de vida do paciente, após a cirurgia. A demarcação deverá ser feita em uma área da pele sem irregularidades, para favorecer a fixação do equipamento coletor, considerando a mobilidade, conforto, tipo físico, configuração do abdómen, capacidades motoras e atividades diárias do paciente. Este é um procedimento crítico a ser realizado no pré-operatório, destacando-se como um dos aspectos mais importantes do processo de reabilitação do estomizado(29).

Com relação às comorbidades e antecedentes familiares dos participantes deste estudo, evidenciou-se que grande parcela dos entrevistados apresentou algum tipo de comorbidade, a hipertensão arterial sistêmica e diabetes. Os dados corroboram com outro estudo que retrata que nos últimos anos as mudanças na sociedade advindas do declínio da taxa de natalidade, da urbanização e maior longevidade populacional, são decorrentes do aumento das doenças crônicas não transmissíveis (DCNT) ${ }^{(19)}$.

Em relação ao tipo de equipamento coletor utilizado, o estudo mostrou que a maioria dos estomizados fazia uso do equipamento de uma peça. Estes achados confirmam o que traz a literatura, em que a maioria dos estomizados faz uso de dispositivos de uma peça, apesar de os dispositivos de duas peças serem os mais indicados, uma vez que favorecem a higienização e facilitam o manuseio. A importância de haver uma variedade de equipamentos disponíveis aos pacientes detona um favorecimento da sua adaptação e incorporação à sua nova situação de vida e a reinserção social mais rapidamente, além de facilidade de manuseio e conforto ${ }^{(30)}$.

É relevante afirmar que os participantes deste estudo apresentaram diversas manifestações clínicas, que os levaram ao diagnóstico da doença de base. Entre as principais queixas elencadas estavam a cólica intestinal, constipação e melena. Como principais manifestações clínicas do diagnóstico do câncer colorretal estão alterações no hábito intestinal, como a mudança na frequência de evacuação, calibre das fezes, presença de sangue nas fezes, anemia, dor abdominal e flatulência. No câncer de reto há, geralmente, queixa de tenesmo, dor retal ou sangramento anal ${ }^{(31)}$.

\section{CONCLUSÃO}

O perfil sociodemográfico dos pacientes em pós-operatório de confecção de EIE es: em sua maioria, do sexo feminino, de cor parda, de 50 a 69 anos, de religião católica, da área urbana, com renda mensal de até um salário mínimo e que estuda até o ensino fundamental incompleto. 
O perfil clínico dos pacientes com estomas intestinais es: em sua maioria, tem Neoplasia do reto, com colostomia eletiva, que não foi demarcada previamente, com presença de comorbidades e antecedentes de câncer na família.

Respeito ao equipamento coletor utilizado, a maioria faz uso do equipamento de uma peça, com uma boa adesão do dispositivo à pele e que tem uma EIE funcionante. A cólica intestinal é a queixa relativas ao procedimento de confecção de EIE mais comum.

Neste contexto, a assistência de enfermagem aos pacientes estomizados exige uma participação ativa do profissional enfermeiro, principalmente no processo de reabilitação e ressocialização, com abordagem individualizada voltadas às dificuldades e necessidades e ao perfil sociodemográfico, considerando as diversos níveis de cognição.

Entre as limitações deste estudo, pode-se destacar a dificuldade de feedback por boa parcela dos pacientes, que demonstravam insegurança e dificuldade em falar sobre determinados assuntos, por constrangimento com a nova condição de saúde.

Para tanto, sugere-se estudos nesta área, com uma abordagem qualitativa, que desvelem a natureza subjetiva desses pacientes e suas necessidades reais para que a assistência de enfermagem possa ser mais diretiva e eficaz para a clientela.

\section{REFERÊNCIAS}

1. Simon BS, Schimith MD, Silveira CL, Budó MLD, Silva MEN, Garcia RP. Configuration of the assistance network to people with ostomy: interface of continuing care. J Nurs Health. 2014; 4(1): 65-76.

2. Coelho AR, Santos FS, Dal Poggetto MT. Stomas changing lives: facing the illness to survive. REME [Internet]. 2013 Abr-Jun [citado 10 ago 2017]; 17(2): 268-77. Disponível em: http://www.reme.org.br/artigo/detalhes/649Stomaschanginglives:

3. Luz MHBA, Andrade DS, Amaral HO, Beze- rra SMG, Benício CDAV, Leal ACA. Characterization of patients submitted to intestinal stomas in a public hospital of Teresina-PI, Brazil. Texto contexto-enferm. 2009; 18(1): 140-46.

4. Diniz IV, Campos MGCA, Vasconcelos JMB, Martins DL, Maia FSB, Caliri MHL. Bolsa de colostomia ou sistema oclusor: vivência de colostomizados. Rev. Estima [Internet]. 2013 [citado 15 fev 2017]; 11(2). Disponível em: https://www.revistaestima.com.br/index. php/estima/article/view/84

5. Cardoso DBR, Almeida CE, Santana ME, Carvalho DS, Sonobe HM, Sawada NO. Sexualidade de pessoas com estomias intestinais. Rev RENE [Internet]. 2015 jul-ago [citado $07 \mathrm{dez}$ 2018] 16(4): 576-85. Disponível em: https:// www.redalyc.org/html/3240/324041519015/

6. Instituto Nacional do Cancer (INCA). Estimativa 2018 - Incidência de câncer no Brasil/ Instituto Nacional de Câncer [Internet]. Rio de Janeiro: INCA; 2017 [citado 07 dez 2018]. 128 p. Disponível em: https://www.inca.gov. br/sites/ufu.sti.inca.local/files//media/document//estimativa-incidencia-de-cancer-nobrasil-2018.pdf.

7. Matsubara MGS, Villela DL, Hashimoto SY, Reis HCS, Saconato RA, Denardi UA et al. Feridas e estomas em oncologia: uma abordagem interdisciplinar. São Paulo: Lemar; 2012. $275 \mathrm{p}$.

8. Mauricio VC, Oliveira NVD, Lisboa MTL. O enfermeiro e sua participação no processo de reabilitação da pessoa com estoma. Esc. Anna Nery. 2013; 17(3): 416-22.

9. Barros ALBL. Anamnese e exame físico Avaliação Diagnostica de enfermagem no adulto. Porto Alegre: Artmed; 2016. 464 p.

10. BRASIL. Ministério da Saúde. Conselho Nacional de Saúde. Diretrizes e normas regulamentadoras sobre pesquisa envolvendo seres humanos. Resolução no 466, de 12 dez 2012 [Internet]. Brasília-DF, 2012 [citado 10 jan 2017]. Disponível em: http://bvsms.saude.gov.br/bvs/saudelegis/cns/2013/ res0466_12_12_2012.html.

11. BRASIL. Ministério da Saúde. Departamento 
de Informática do SUS. Sistema de informações sobre mortalidade [Internet]. Brasília, DF: Ministério da Saúde. 2017 [citado $07 \mathrm{dez}$ 2018]. Disponível em: http://www.datasus. gov.br.

12. Ardigo FS, Amante LN. Knowledge of the professional about nursing care of people with ostomies and their families. Texto contexto-enferm. 2013; 22(4): 1064-71.

13. Instituo Brasileiro de Geografia e Estatística. Projeção da população do Brasil e Unidades da Federação por sexo e idade para o período 2000-30 [Internet]. Rio de Janeiro: IBGE; 2017 [citado 06 dez 2018]. Disponível em: https://www.ibge.gov.br/home/estatistica/ populacao/projecao_da_populacao/2013/ default.shtm.

14. Boaventura AP, Vedovato CA, Santos FFD. Profile of the oncologic patients treated at the emergency unit. Cienc. enferm. 2015; XXI(2): 51-62.

15. Luz Ad, Araújo Luz MB. Perfil de pacientes ostomizados atendidos por la estrategia salud de la familia. Rer Cubana Enfermer [Internet]. 2015 [citado 7 dic 2018]; 30(2). Disponível em: http://www.revenfermeria.sld.cu/ index.php/enf/article/view/668.

16. Menezes LCG, Guedes MVC, Oliveira RM, Oliveira SKP, Meneses LST, Castro ME. Selfcare practice of ostomy patients: contributions of the Orem's theory. Rev. RENE. 2013; 14(2): 301-10.

17. Araújo SNM, Luz MHBA, Rocha SS, Silva GRF, Duarte MR, Sandes NM. Obesidade infantil: conhecimentos e práticas de enfermeiros da Atenção Básica. Enfermagem em Foco. 2012; 3(3): 139-42.

18. Paula MAB, Paula PR, Cesaretti IUR. Estomaterapia em foco: e o cuidado especializado. São Caetano do Sul, SP: YENDIS; 2014. $456 \mathrm{p}$.

19. Macêdo MS, Nogueira LT, Luz MHBA. Perfil dos estomizados atendidos em hospital de referência em Teresina. Rev. Estima [Internet]. 2005 [citado 10 abr 2017]; 3(4): Disponível em: https://www.revistaestima.com.br/index.php/estima/article/view/15/0
20. Associação Brasileira de ostomizados - ABRASO/Carvalheira C, coord. Cartilha do homem ostomizado I. Rio de Janeiro: ABRASO; 2004. $24 \mathrm{p}$.

21. Sasaki VDM, Pereira APS, Ferreira AM, Pinto $\mathrm{MH}$, Gomes JJ. Health care service for ostomy patients: profile of the clientele. J Coloproctol (Rio J.). 2012; 32(3): 232-239.

22. Santos VLCG. Aspectos Epidemiológicos dos Estomas. Rev Estima [Internet]. 2007 [citado 04 mar 2017]; 5(1). Disponível em: https:// www.revistaestima.com.br/index.php/esti$\mathrm{ma} /$ article/view/207

23. Martins PAF, Alvim NAT. Perspectica educativa do cuidado de enfermagem sobre manutenção da estomia de eliminação. Rev Bras Enfer. 2011; 64(2): 322-27.

24. Carvalho SORM, Budó MLD, Silva MM, Alberti GF, Simon BS. "With some care, wecan go on": experiences of people with ostomy. Texto contexto-enferm. 2015; 24(1): 279-87.

25. Miranda SM, Luz MHBA, Sonobe HM, Andrade EMLR, Moura ECC. Sociodemographic and Clinic Characterization of People with Ostomy in Teresina. Rev Estima; 2016. 14(1): 29-35.

26. Luz ALA, Luz MHBA, Antunes A, Oliveira GS, Andrade EMLR, Miranda SM. Perfil de pacientes estomizados: revisão integrativa da literatura. Cultura de los cuidados [Internet]. 2014 [citado 15 abr 2017]; 18(39): 115-23. Disponível em: https://rua.ua.es/dspace/bitstream/10045/40073/1/Cultura_Cuidados_39_13.pdf

27. Batista MRFF, Rocha FC, Silva DM, Júnior FJ. Self-image of clients with colostomy related to the collecting bag. Rev Bras Enferm. 2011; 64(6): 1043-7.

28. Salome GM, Santos L, Cabeceira H, Panza A, Paula M. Knowledge o funder graduate nursing course teachers on the prevention and care of peristomal skin. J Coloproctol (Rio J.). 2014; 34(4): 224-30.

29. Miranda SM, Nascimento CMFS, Luz MHBA, Andrade EMLR, Luz ALA, Torres CRD. Viver com estomia: contribuições para assistência de Enfermagem. Rev. Estima. 2014 [citado 
05 mar 2017]. 12(3). Disponível em: https:// www.revistaestima.com.br/index.php/esti$\mathrm{ma} /$ article/view/94.

30. Bonill-de-las-Nieves C, Celdrán-Mañas M, Hueso-Montoro C, Morales-Asencio JM, Rivas-Marín C, Fernández-Gallego MC. Living with digestive stomas: strategies to cope with the new bodily reality. Rev Lat Am Enfermagem. 2014; 22(3): 394-400.

31. Silva J, Sonobe HM, Buetto LS, Santos MG, Lima MS, Sasaki VDM. Teaching strategies for self-care of the intestinal stoma patients. Rev. RENE. 2014; 15(1): 166-73. 